Check for updates

Cite this: RSC Adv., 2017, 7, 28677

\title{
Control of the compensating defects in Al-doped and Ga-doped ZnO nanocrystals for MIR plasmonics $\dagger$
}

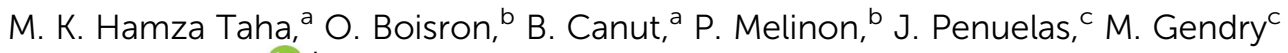 \\ and B. Masenelli (D) *a
}

In degenerate semiconductor nanoparticles, the tuning range of the plasmon resonance is directly controlled by the electron gas concentration and thus by the dopant activation. Here, we investigate the improvement of the dopant activation in $\mathrm{ZnO}$-based nanocrystals for mid IR plasmonics. For that purpose, we have synthesized Al-doped and Ga-doped ZnO nanocrystals in O-rich and O-poor environments. We show that the free carrier concentration can be doubled for the samples grown in an O-poor environment. Accordingly, the plasmon resonance shifts from $5 \mu \mathrm{m}$ to $3.1 \mu \mathrm{m}$. In analogy with previous results from $\mathrm{Ga}$-doped $\mathrm{ZnO}$ thin films, we discuss the effect of the possible reduction of the concentration of acceptor-like complexes such as $\mathrm{Al}_{\mathrm{Zn}}-\mathrm{V}_{\mathrm{Zn}}$ and $\mathrm{Al}_{\mathrm{Zn}}-\mathrm{O}_{\mathrm{i}}$ (resp. $\mathrm{Ga}_{\mathrm{Zn}}-\mathrm{V}_{\mathrm{Zn}}$ and $\mathrm{Ga}_{\mathrm{Zn}}-\mathrm{O}_{\mathrm{i}}$ ) on the activation improvement. Besides, whether long or rapid, thermal annealing does not improve the compensation ratio. Consequently, the control of $O$ content during synthesis remains the most valuable tool to achieve the highest dopant activation in doped $\mathrm{ZnO}$ nanocrystals.

Received 30th March 2017

Accepted 18th May 2017

DOI: 10.1039/c7ra03697c

rsc.li/rsc-advances plasmon resonance wavelength can be tuned from about $1 \mu \mathrm{m}$ to $5 \mu \mathrm{m}$. However, when the doping level reaches degeneracy, the activation of dopant is reduced, as is evidenced by Brouwer's diagram. ${ }^{12}$ Generally, much less than $1 / 2$ of the dopant atoms introduced effectively contribute to the free electron gas. Typical values of the dopant activation usually range between $10 \%$ and $50 \%$. This is particularly true for $\mathrm{Ga}$ and $\mathrm{Al}$ dopants in $\mathrm{ZnO}$ nanocrystals or for $\mathrm{Sn}$ in indium tin oxide (ITO) particles. ${ }^{\mathbf{1 3 , 1 4}}$ The issue of incomplete dopant activation has been addressed first by groups working on transparent conductive oxide (TCO) thin films. The dopant efficiency is reduced because of the formation of compensating centers. For instance, in the case of Sn doped indium oxide, ${ }^{15}$ complexes between substitutional tin $\left(\mathrm{Sn}_{\mathrm{In}}\right)$ and oxygen interstitials $\left(\mathrm{O}_{\mathrm{i}}\right)$ are increasingly stable and act as acceptor centers, hence compensating the n-doping of Sn. The same kind of mechanism has been evoked in Ga-doped $\mathrm{ZnO}$ thin films ${ }^{16}$ with the formation of $\mathrm{Ga}_{\mathrm{Zn}}-\mathrm{O}_{\mathrm{i}}$ and $\mathrm{Ga}_{\mathrm{Zn}}-\mathrm{V}_{\mathrm{Zn}}$ acceptor complexes. Because of the required presence of defects such as $\mathrm{Zn}$ vacancies $\left(\mathrm{V}_{\mathrm{Zn}}\right)$ or $\mathrm{O}$ interstitials $\left(\mathrm{O}_{\mathrm{i}}\right)$, the formation energy of these complexes depends on the amount of oxygen during the growth. Thus, in O-rich growth conditions, the presence of both $\mathrm{V}_{\mathrm{Zn}}$ and $\mathrm{O}_{\mathrm{i}}$ is expected to be favored and the formation energy of the $\mathrm{Ga}_{\mathrm{Zn}}-\mathrm{O}_{\mathrm{i}}$ and $\mathrm{Ga}_{\mathrm{Zn}}-\mathrm{V}_{\mathrm{Zn}}$ acceptor complexes is low. On the contrary, in O-poor growth conditions, the stabilization of the acceptor complexes is less likely. This mechanism has been evoked to explain the improved electric conductivity of TCO grown in O-poor conditions. It is thus appealing to study the impact of oxygen on the electronic and 
optical properties of MIR nanocrystals, and hence on the tuning of the plasmon resonance.

In this work, we investigate the influence of oxygen pressure during the synthesis on the plasmon resonance in Al-doped (AZO) and Ga-doped (GZO) ZnO nanocrystals. We report on the physical synthesis of uncapped $\mathrm{ZnO}$ nanocrystals doped at $3 \%$ with either $\mathrm{Al}$ or $\mathrm{Ga}$ by an adiabatic plasma expansion in high vacuum. The doping level of $3 \%$ was chosen as an optimal compromise between an intense LSPR and a good reproducibility of the samples. Indeed, we have observed in a previous work ${ }^{17}$ that for doping levels lower than $6 \%$, the nanocrystals are homogeneous in doping and thus are below the solubility limit of $\mathrm{Al}$ or $\mathrm{Ga}$ within our synthesis process while exhibiting clear and reproducible LSPR. Since the nanocrystals had uncapped surfaces, they tended to spontaneously self-organize through the oriented attachment (OA) process. Doped-ZnO nanocrystals have been synthesized in O-poor as well as in $\mathrm{O}$ rich conditions. We have succeeded in modulating the plasmon resonance from $5 \mu \mathrm{m}$ to about $3.1 \mu \mathrm{m}$ by synthesizing the nanocrystals in an O-poor environment. Correspondingly, the resulting free carrier density has been increased significantly. We discuss about the possible relation between this increase in free carrier density and the concentration reduction of acceptor defects and complexes such as $\mathrm{Al}_{\mathrm{Zn}}-\mathrm{O}_{\mathrm{i}}$ and $\mathrm{Al}_{\mathrm{Zn}}-\mathrm{V}_{\mathrm{Zn}}$ (resp. $\mathrm{Ga}_{\mathrm{Zn}}-\mathrm{O}_{\mathrm{i}}$ and $\mathrm{Ga}_{\mathrm{Zn}}-\mathrm{V}_{\mathrm{Zn}}$ as seen in Ga-doped $\mathrm{ZnO}$ thin films). Next we investigate the impact of a post-deposition annealing to further improve the dopant activation. Contrary to what is observed for thin films, thermal treatment is detrimental to the plasmon of the nanocrystals, questioning the metastability of the dopants.

\section{Methods}

The nanocrystals have been synthesized by the low energy cluster beam deposition (LECBD) technique described in. ${ }^{\mathbf{1 7 , 1 8}} \mathrm{A}$ plasma is obtained from a target which is ablated using a pulsed (10 Hz repetition rate) doubled (532 nm; $100 \mathrm{~mW}$ ) YAG: Nd laser. The $3 \% \mathrm{Al}(\mathrm{Ga})$ doped $\mathrm{ZnO}$ pellet target consists of a mixture of $\mathrm{ZnO}$ and $\mathrm{Al}_{2} \mathrm{O}_{3}\left(\mathrm{Ga}_{2} \mathrm{O}_{3}\right)$ (99.999\% pure each) microscopic powders sintered and annealed at $800{ }^{\circ} \mathrm{C}$ in air for $10 \mathrm{~h}$. A constant flux of buffer gas is used to quench the plasma. The gas flux was a mixture of $75 \% \mathrm{He}$ and $25 \% \mathrm{O}_{2}$ for the AZO nanocrystals obtained in O-rich conditions. For the O-poor synthesis, no $\mathrm{O}_{2}$ gas was added to He. After the ablation of the target, the gas induces stabilization of nucleation embryos (dimers and trimers). Subsequently the mixture of the plasma species and buffer gas is expanded through a micrometer nozzle while leaving the nucleation chamber at 20 mbar and reaching the deposition chamber at $10^{-7}$ mbar. During this adiabatic expansion, atoms from the plasma aggregate according to an accretion process; at that point the formation of the clusters is achieved. The resulting nanocrystals forming a supersonic jet are deposited on silicon substrates or TEM grids. Despite their high velocity, the atomic kinetic energy in the nanocrystals is lower than the atomic binding energy. Therefore, the nanocrystals do not fragment during deposition. The films resulting from the deposition of the nanocrystals are typically several hundreds of nm thick and foam-like, with a porosity estimated to be about $70 \%$ according to the $3 \mathrm{D}$ percolation theory.

The structure of the deposited nanocrystals was investigated by transmission electron microscopy (TEM) and X-ray diffraction (XRD). High resolution TEM images were acquired on a JEOL $2010 \mathrm{~F}$ operating at $200 \mathrm{kV}$, equipped with a Gatan Orius 100 CCD camera. XRD diffractograms were acquired on a Rigaku Smartlab diffractometer using the $\mathrm{Cu} \mathrm{K}_{\alpha 1}$ radiation $(\lambda$ $=1.54 \AA$ ), the incident beam was fixed at a grazing angle of $0.4^{\circ}$ in order to increase the surface diffraction. The elemental composition of the whole nanocrystal films has been measured by Rutherford Backscattering Spectrometry (RBS) using a ${ }^{4} \mathrm{He}^{++}$ ion beam accelerated at $5 \mathrm{MeV}$ delivered by the van de Graaff accelerator of the Nuclear Physics Institute of Lyon (IPNL). A 13 $\mathrm{keV}$ resolution implanted junction, set at an angle of $172^{\circ}$ with respect to the beam axis, has been used to detect the backscattered particles. Chemical states of the constituents have been analyzed by X-ray Photoelectron Spectroscopy (XPS), using an $\mathrm{Al}$ anode. The spectra have been calibrated by using the $\mathrm{C} 1 \mathrm{~s}$ level of adventitious carbon species as standard references with a nominal value of $285.0 \mathrm{eV}$. Eventually, the plasmon resonances have been probed by Fourier Transform Infrared Spectroscopy (FTIR) in the reflection mode.

\section{Results}

The LECBD technique has been previously used to synthesize undoped and $\mathrm{Ga}$ doped $\mathrm{ZnO}$ nanocrystals. ${ }^{17,18}$ As in these previous cases, the Al-doped and Ga-doped nanocrystals synthesized in both O-rich and O-poor environments are crystallized in the wurtzite structure. This is confirmed by TEM analysis in the high resolution mode and shown in Fig. 1. The average size distribution of the nanocrystals (estimated over 100 particles) is $5.6 \pm 0.3 \mathrm{~nm}$.

Fig. 2 shows the XRD diffractograms of AZO nanocrystal films. The peaks correspond to the wurtzite structure with no additional peak indicating the absence of any other crystalline phase (see Fig. S2 of ESI $\dagger$ for large scan diffractogram). However, the average size of the crystalline domains, deduced using Scherrer's equation and assuming perfect crystals without defects, is $29 \pm 2 \mathrm{~nm}$ for all samples. This discrepancy has already been pointed out and reported for pure $\mathrm{ZnO}^{18}$ and $\mathrm{Ga}$ doped $\mathrm{ZnO}$ nanocrystals ${ }^{\mathbf{1 7}}$ produced by the LECBD technique. It results from the oriented attachment (OA) of the initial uncapped nanocrystals (see Fig. S3 of ESI $\dagger$ ). The nanocrystals tend to reduce the surface energy of their facets through epitaxial attachment. The influence of the OA on MIR plasmon resonance has been studied intensively in. ${ }^{17}$ We have shown that it essentially leads to the inhomogeneous broadening of the plasmon resonance but has almost no effect on the dopant compensation. Moreover, the diffractograms do not show any shift of the peak positions. In AZO nanocrystals synthesized by wet chemical route, a slight increase of the peak position, corresponding to a small shrinkage of the lattice, has been reported. ${ }^{19} \mathrm{Al}$ having an ionic radius $(50 \mathrm{pm})$ smaller than that of $\mathrm{Zn}$ (74 pm), this shrinkage has been considered as a confirmation of the substitution of $\mathrm{Zn}$ with $\mathrm{Al}$. If this statement holds, we 


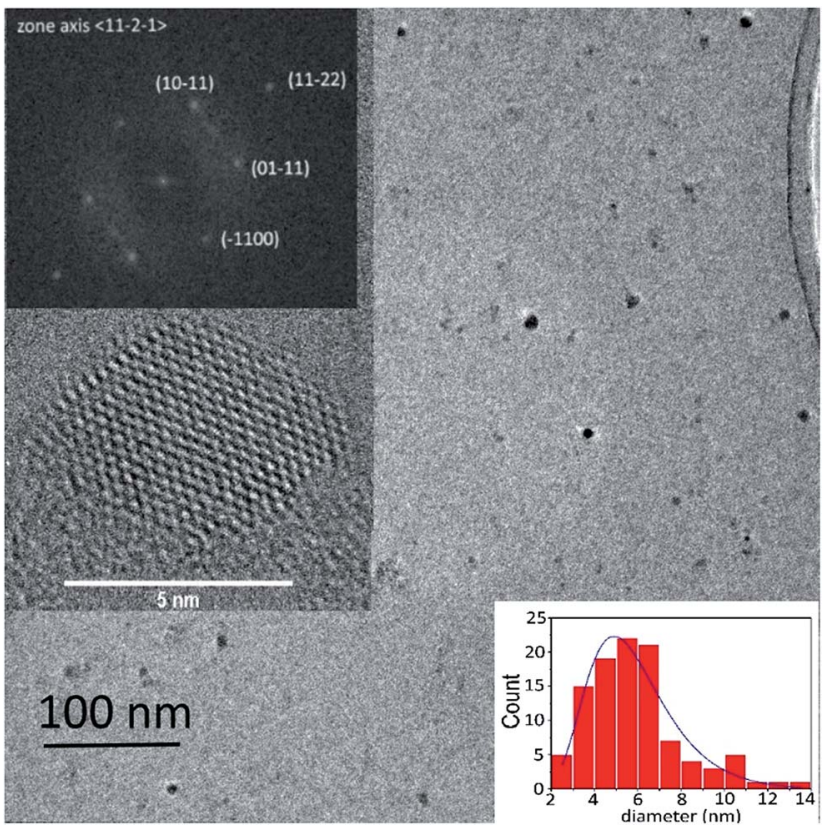

Fig. 1 Low magnification TEM image of AZO nanocrystals. Inset: high resolution TEM image of an AZO nanocrystal, doped at $3 \%$ and the corresponding FFT, indicating the wurtzite structure of the nanocrystal. The size distribution of the nanocrystals is also shown (lower inset) revealing a mean size of $5.6 \mathrm{~nm}$. The solid line is a fit to the data with a log-normal distribution, typical of the accretion process involved in the LECBD synthesis.

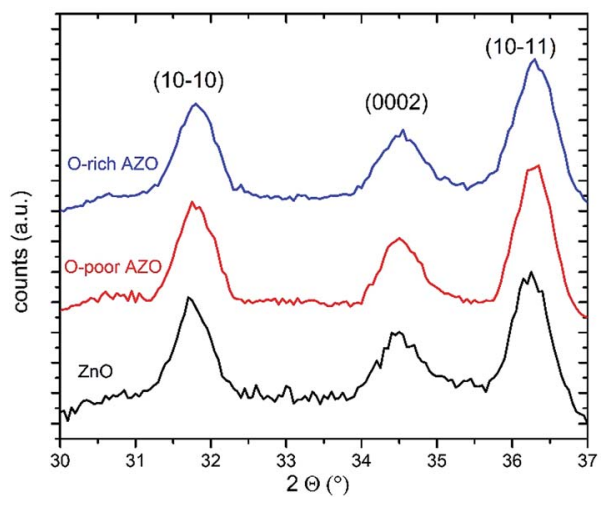

Fig. 2 Diffractograms of the $\mathrm{ZnO}$ nanocrystal sample and of O-rich and O-poor AZO nanocrystal samples with $3 \%$ Al content.

can infer that, in our nanocrystals, not all the $\mathrm{Al}$ atoms are incorporated on substitutional sites. Nevertheless, the incorporation of $\mathrm{Al}$ (or $\mathrm{Ga}$ ) in our nanocrystal films has been confirmed by RBS. The measured content of $\mathrm{Al}$ in AZO and Ga in GZO nanocrystals, whether obtained in O-rich or O-poor environment, is $3 \%$ in atomic concentration with respect to $\mathrm{Zn}$.

The effect of synthesizing nanocrystals in O-poor and O-rich environments on the IR plasmon properties of the resulting films was investigated by FTIR reflectance measurements. The plasmon peaks of the $3 \%$ AZO and GZO nanocrystals synthesized in O-rich and O-poor environments are shown in Fig. 3. As
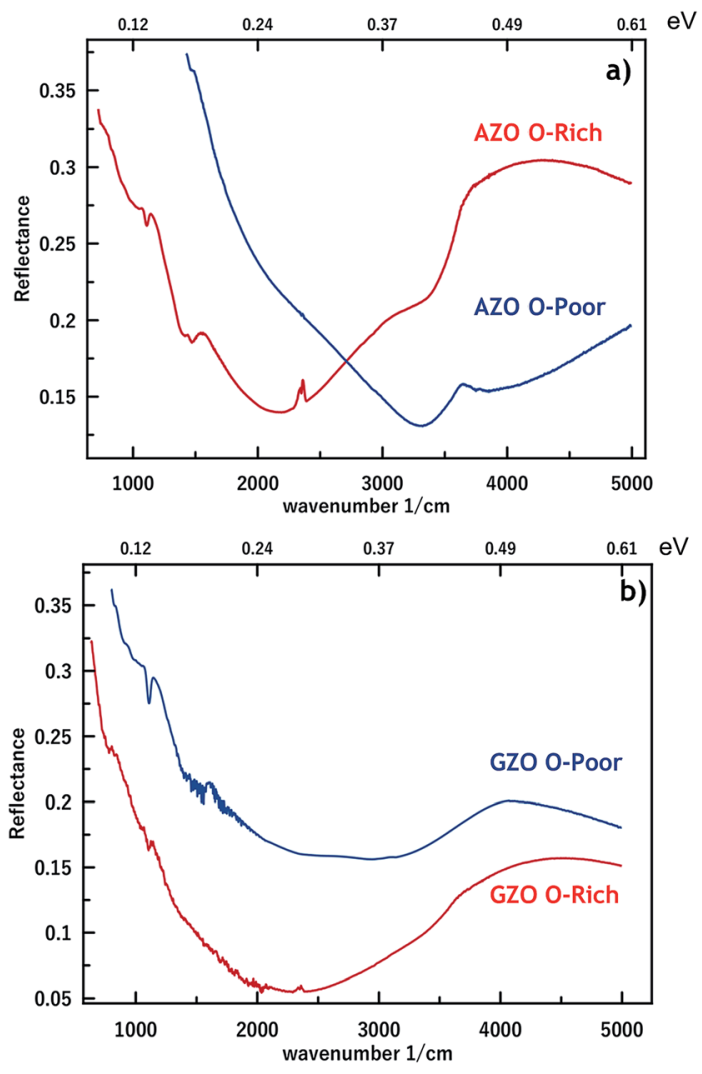

Fig. 3 FTIR reflectance spectra of (a) AZO and (b) GZO nanocrystal films synthesized in oxygen rich (O-rich) and oxygen poor (O-poor) conditions deposited on Si substrates.

expected, the plasmon peaks are characterized by large broadening. This broadening is a common feature of doped nanocrystals. ${ }^{6,13,14,17}$ It is related to several distinct causes, such as the inherent plasmon damping (in Drude's model), which leads to the homogeneous broadening. It is also related to the inhomogeneity of the nanocrystal size and shape, ${ }^{20,21}$ the dopant segregation among the nanocrystals, the variation of the electron concentration between nanocrystals ${ }^{12}$ and the nanocrystal organization (oriented attachment). The sum of the optical responses of nanocrystals of different sizes, shapes and electron densities gives rise to a broad plasmon peak.

In Fig. 3a, we observe that the plasmon resonances of AZO nanocrystal films resulting from a synthesis in O-rich and $\mathrm{O}$ poor environments peak at $2000 \mathrm{~cm}^{-1}(5 \mu \mathrm{m}$ or $0.25 \mathrm{eV})$ and $3200 \mathrm{~cm}^{-1}(3.1 \mu \mathrm{m}$ or $0.40 \mathrm{eV})$, respectively. For GZO nanocrystals (Fig. 3b), the shift is slightly lower, ranging from 2000 $\mathrm{cm}^{-1}(5 \mu \mathrm{m}$ or $0.25 \mathrm{eV})$ to $2700 \mathrm{~cm}^{-1}(3.75 \mu \mathrm{m}$ or $0.33 \mathrm{eV})$. From the observed plasmon resonances, we deduce the free electron density according to Drude's model. The frequency of the bulk plasmon resonance $\omega_{\mathrm{p}}$ is related to the electron density $n$ through the following expression:

$$
\omega_{\mathrm{p}}=\sqrt{\frac{n e^{2}}{\varepsilon_{0} \varepsilon_{\mathrm{r}} m^{*}}}
$$


with $e$ the electron charge, $m^{*}$ the effective mass of free electrons in $\mathrm{ZnO}, \varepsilon_{\mathrm{o}}$ the permittivity of vacuum and $\varepsilon_{\mathrm{r}}$ the relative permittivity of $\mathrm{ZnO}$. The localized plasmon induced in nanocrystals dispersed in a surrounding medium (i.e. foam-like thinfilm) with a permittivity $\varepsilon_{\mathrm{m}}$ is:

$$
\omega_{\mathrm{sp}}=\sqrt{\frac{\omega_{\mathrm{p}}{ }^{2}}{\varepsilon_{\infty}+2 \varepsilon_{\mathrm{m}}}}
$$

where $\varepsilon_{\infty}$ is the high frequency permittivity of AZO or GZO (assumed to be equal to 3.7, the $\mathrm{ZnO}$ value). $\varepsilon_{\mathrm{m}}$ is deduced from the Bruggeman model since it is the most suited to describe our samples, where nanocrystals are connected and in contact with each other forming a random network with $70 \%$ porosity (as explained before). The effective medium surrounding each nanocrystal is thus a mixture of $70 \%$ of air and $30 \%$ of other identical nanocrystals. Using the expression of $\omega_{\text {sp }}$, we extracted the electron density values. The free carrier concentration for AZO nanocrystals from O-rich environment is $2 \times 10^{20} \mathrm{~cm}^{-3}$, while it is $4.1 \times 10^{20} \mathrm{~cm}^{-3}$ for AZO nanocrystals from the O-poor environment. For GZO nanocrystals, the carrier concentrations are $2 \times 10^{20} \mathrm{~cm}^{-3}$ and $3.4 \times 10^{20} \mathrm{~cm}^{-3}$, respectively. In other words, these quantities represent roughly 700 and up to 1600 free electrons per nanocrystal. We have to concede that these values of free carrier concentrations are approximations (overestimations). This is a consequence of the inherent error induced by eqn (2) for degenerate semiconductor nanocrystals. ${ }^{18}$ We observe that the carrier density in AZO nanocrystal films synthesized in O-poor conditions has doubled as compared to the one prepared in O-rich conditions and has been multiplied par a factor of 1.6 for GZO nanocrystal films. Accordingly, the fraction of active dopants amounts roughly to $1 / 6$ in the O-rich AZO nanocrystals and to $1 / 3$ in the O-poor ones (1/6 in the O-rich GZO nanocrystals and about 1/4 for O-poor GZO nanocrystals). The improvement trend is common to AZO and GZO but the improvement magnitude is somewhat smaller for Ga doping.

Synthesizing AZO or GZO nanocrystal films in O-poor conditions is conclusively beneficial for the improvement of the free electron density. This observation has been demonstrated for $\mathrm{GZO}^{16,22}$ or $\mathrm{AZO}^{23}$ thin films. As mentioned in the introduction, in the latter case, the improvement has been assigned to the reduced concentration of the $\mathrm{Ga}_{\mathrm{Zn}}-\mathrm{V}_{\mathrm{Zn}}$ or $\mathrm{Ga}_{\mathrm{Zn}^{-}}$ $\mathrm{O}_{\mathrm{i}}$ acceptor complexes. Demchenko et al. ${ }^{\mathbf{1 6}}$ have computed the formation energy of these complexes and shown that in O-rich conditions, when the Fermi level is high, as in the case of degenerate $\mathrm{ZnO}$, it is lower than that of the donor $\mathrm{Ga}_{\mathrm{Zn}}$. Actually, the most stable and thus most likely complex is $\mathrm{Ga}_{\mathrm{Zn}}-\mathrm{V}_{\mathrm{Zn}}$. In O-poor conditions, the formation energy of both complexes is higher by $6.70 \mathrm{eV}$ making them less likely to exist. Hence, the compensating effect is reduced. This is a common feature in $\mathrm{Al}$ or Ga doped $\mathrm{ZnO}$ thin films. Moreover, other kinds of acceptor defects have been pointed out. At odd with the DFT calculations from Demchenko et al. which do not predict $\mathrm{V}_{\mathrm{Zn}}$ as a stable defect, Look et al. have noticed that, in films grown with pulsed laser ablation, $\mathrm{V}_{\mathrm{Zn}}$ can compensate partially the donors. ${ }^{24}$ Nevertheless, this study also points towards the role of $\mathrm{Zn}$ deficiencies, and thus indirectly to the $\mathrm{O}$ pressure during the growth, since a large $O$ pressure is expected to favor the presence both $\mathrm{V}_{\mathrm{Zn}}$ and $\mathrm{O}_{\mathrm{i}}$. In Ga-doped $\mathrm{ZnO}$ thin films, the compensation ratio was reduced from $90 \%$ to less than $30 \%$ (ref. 14 ) or set at $57 \%$ by controlling the synthesis ambient. In our AZO nanocrystal films the compensation ratio is reduced from $83 \%$ to $65 \%$. Since we experience exactly the same behavior related to the $\mathrm{O}$ conditions of growth, we believe that in our system, acceptors either related to $\mathrm{V}_{\mathrm{Zn}}$ or $\mathrm{O}_{\mathrm{i}}$ are involved, possibly the $\mathrm{Ga}_{\mathrm{Zn}}-\mathrm{V}_{\mathrm{Zn}}$ or $\mathrm{Ga}_{\mathrm{Zn}}-\mathrm{O}_{\mathrm{i}}$ acceptor complexes.

In order to support this hypothesis, we have analyzed the chemical states of $\mathrm{O}$ within the nanocrystals by XPS. The O1s levels of O-rich and O-poor AZO nanocrystals are shown in Fig. 4. Both spectra show two distinct contributions labelled O1 and $\mathrm{O} 2$. The $\mathrm{O} 1$ contribution, peaking at $530.6 \mathrm{eV}$, is ascribed to $\mathrm{O}^{2-}$ ions in a well-crystallized wurtzite environment, in accordance with ref. 25 and 26. The second contribution $\mathrm{O} 2$, peaking at $532 \mathrm{eV}$ is assigned to $\mathrm{O}$ from hydroxide groups or to $\mathrm{O}$ in an $\mathrm{O}$ deficient environment. $\mathrm{O} 2$ line is thus and indirect signature of $\mathrm{O}$ vacancies $\left(\mathrm{V}_{\mathrm{O}}\right)$. The ratio of the intensities of the $\mathrm{O} 1$ and $\mathrm{O} 2$ lines changes from 1.6 for the O-rich nanocrystals to 0.6 for the $\mathrm{O}$-poor ones. The $\mathrm{OH}$ content being identical in both samples, the change of the $\mathrm{O} 1 / \mathrm{O} 2$ ratio indicates the reduction of $\mathrm{O}$ content in the nanocrystals synthesized in O-poor conditions.
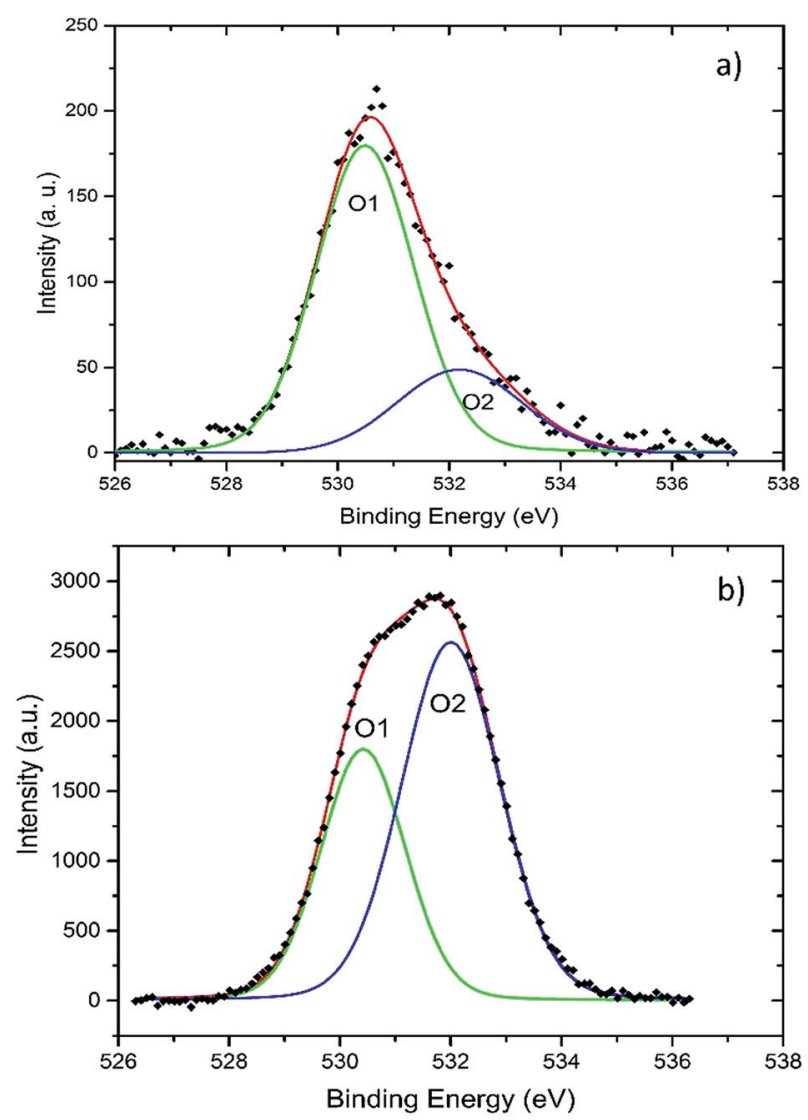

Fig. 4 XPS spectra of the O1s level for 3\% AZO nanocrystals synthesized in O-rich conditions (a) and O-poor conditions (b). The experiment results correspond to symbols. The $\mathrm{O} 1$ and $\mathrm{O} 2$ contributions are shown as the result of their sum (solid line). 
Accordingly, the presence of $\mathrm{O}_{\mathrm{i}}$ and $\mathrm{V}_{\mathrm{Zn}}$ is expected to be drastically reduced, as is the presence of related compensating centers.

Even though we managed to reduce the acceptor complexes concentration, we note that our samples still have other compensating defects. Indeed, the presence of uncapped surfaces, of grain boundaries and twin boundaries resulting from the oriented attachment may play also a role. Embedding the AZO (or GZO) nanocrystals in a matrix leading to a clean and passivated surface of the nanocrystals might reduce significantly the acceptor-like defects ( see $^{27}$ ). Moreover, controlling the position of the donor centers among the nanocrystals could also be beneficial, as revealed by Milliron et al. for nanocrystals ${ }^{15}$ and by Look et al. for thin films. ${ }^{28}$ Eventually, we have to consider the possibility that the dopants do not go on the proper sites. In the drastically kinetic conditions of our growth process, we cannot discard that some $\mathrm{Al}$ or $\mathrm{Ga}$ atoms are incorporated on $\mathrm{O}$ sites (anti-sites) or interstitial sites. This would lead to metastable acceptor defects. As evidenced previously, the high resolution Xrays diffractograms of pure $\mathrm{ZnO}$ and $3 \% \mathrm{AZO}$ nanocrystals (synthesized in O-rich or O-poor condition) do not show any shift of the peak positions. This may reveal that a significant part of the $\mathrm{Al}$ ions is not incorporated in $\mathrm{Zn}$ sites.

This observation has been made also for thin films and the latter are often subject to post-deposition annealing. Consequently, we have first annealed our 3\% AZO O-rich nanocrystal films in air for 30 minutes at low temperature $\left(50^{\circ} \mathrm{C}\right)$ and high temperature $\left(400^{\circ} \mathrm{C}\right)$. In Fig. 5, we clearly observe that the low temperature annealing has no effect on the LSPR while the high temperature one leads to its complete disappearance. This is in fair agreement with results from Kortshagen et al. ${ }^{27}$ where the disappearance of the LSPR in AZO nanocrystals was observed after a $1 \mathrm{~h}$ annealing at $650{ }^{\circ} \mathrm{C}$. Thus, instead of improving the activation of the dopants, it clearly shows that either the latter are in a metastable state or annealing induces additional compensating centers. Taking this metastability into account and considering that the diffusion length of $\mathrm{Al}$ in $\mathrm{ZnO}$ at high temperature can be as large as few nanometers, we next annealed the $3 \%$ AZO O-rich nanocrystal films by Rapid Thermal Annealing (RTA) in vacuum at moderate temperature $\left(350^{\circ} \mathrm{C}\right)$ for 1 or 2 minutes. This temperature was chosen from studies in the literature showing improvements of degenerate $\mathrm{ZnO}$ close to this value. ${ }^{29-32}$ The results are presented in Fig. $5 \mathrm{~b}$. Here again annealing leads to the annihilation of the LSPR. Our results emphasize a discrepancy between certain studies regarding degenerate $\mathrm{ZnO}$ thin films and our nanocrystals since in thin films a RTA has been shown to improve the dopant activation. ${ }^{16}$ However, for the sake of rigor, we mention that even in degenerate thin films, annealing at moderate temperature $\left(\mathrm{ca} .350{ }^{\circ} \mathrm{C}\right)$ has been show to degrade the dopant concentration and thus the electron gas concentration. ${ }^{33}$ Actually, the annealing atmosphere matters. It seems that annealing in air, oxygen or nitrogen reduces the carrier concentration ${ }^{32,33}$ whereas annealing in reducing atmosphere seems to improve it. $^{34-36}$ To further check the influence of the annealing atmosphere, we have repeated the RTA in a neutral ambient, Ar. The reduction of the electron gas concentration is still observed but
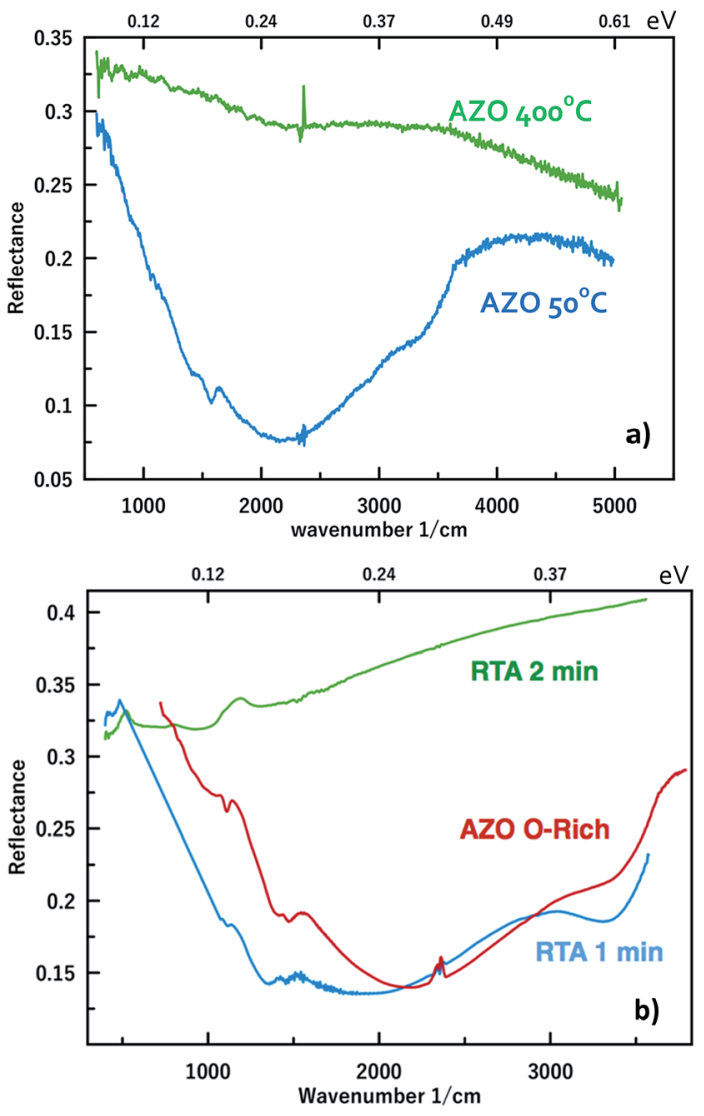

Fig. 5 FTIR reflectance spectra of (a) 3\% AZO nanocrystal films annealed in air at $50{ }^{\circ} \mathrm{C}$ and $400{ }^{\circ} \mathrm{C}$ for 30 minutes and (b) of $3 \% \mathrm{AZO}$ nanocrystal films annealed by RTA at $350{ }^{\circ} \mathrm{C}$ for $1 \mathrm{mn}$ and $2 \mathrm{mn}$.

to a lesser extent (see ESI S4†). Eventually, we have annealed an O-rich $3 \%$ doped AZO nanocrystal sample in $\mathrm{H}_{2}\left(400{ }^{\circ} \mathrm{C}, 30\right.$ min). The MIR reflectance spectrum, presented in (Fig. S5 of ESI $\dagger$ ), does not show any LSPR anymore. This highlights that both mechanisms are involved. The addition of compensator centers is evidenced by the fact that the reduction of the LSPR is larger with an oxidizing ambient. On the other hand, when annealing in an inert atmosphere, the introduction of charged species is less likely. Eventually, the annealing in a reducing atmosphere should provide extra electrons to help saturating the electron traps. However, in the annealing conditions used here, this is not the case. The duration of the annealing seems to matter. The LSPR disappearance thus questions the metastability of the acceptors.

Another important point concerns the homogeneity of the compensation. We have no evidence about its possible variation from nanocrystal to nanocrystal but we can expect at least some stochastic variations. Since it has been shown that variations of the free electron density between different nanocrystals lead to an inhomogeneous broadening of the LSPR, ${ }^{13}$ it would be very interesting to measure the compensating ratio at the nanocrystal scale and see how it can be controlled in order to design nanocrystals with very sharp LSPR. 


\section{Conclusion}

To conclude, we have investigated the improvement of dopant activation in $\mathrm{ZnO}$ nanocrystals for Mid IR plasmonics. First, the influence of the $\mathrm{O}$ amount during the growth on the LSPR of AZO and GZO nanocrystals has been studied. We have synthesized nanocrystal films either in O-rich or O-poor environment. While the $\mathrm{Al}$ (or Ga) content is not affected, the carrier density is doubled, varying from $2 \times 10^{20} \mathrm{~cm}^{-3}$ for the O-rich synthesis to $4 \times 10^{20} \mathrm{~cm}^{-3}$ for the O-poor one. Accordingly, the compensation ratio decreases from $83 \%$ to $65 \%$. In analogy with previous calculations and observations on Ga-doped and Al-doped $\mathrm{ZnO}$ nanocrystal films, we propose that the increase in the carrier density is related to the concentration reduction of acceptor-like defects and complexes such as $\mathrm{Al}_{\mathrm{Zn}}-\mathrm{V}_{\mathrm{Zn}}$ and $\mathrm{Al}_{\mathrm{Zn}}-\mathrm{O}_{\mathrm{i}}$. Despite the improvement of the compensating ratio the carrier density absolute value remains rather low, pointing to the presence of other compensating centers. Contrary to what has been observed for thin films, the removal of the latter cannot be achieved by post-synthesis thermal annealing. Instead, the annealing questions the metastability of the dopants within the nanocrystals and is accompanied with the disappearance of the LSPR. Thus, the control of the synthesis conditions, and among them the amount of $\mathrm{O}$, is of prime importance to enhance the activation of dopants in degenerate $\mathrm{ZnO}$ nanocrystals.

\section{Acknowledgements}

This works was financed through the GaZON project ANR-15CE-09-004-01. The authors are indebted to Prof. K. MasenelliVarlot and the CLYM platform for TEM analysis. B. M. wishes to thank Dr Dave Rodgers from Nanovation for fruitful discussion. The authors are very grateful to the technical staff of the ANAFIRE platform of Institute of Nuclear Physics of Lyon (IPNL) for their help during the experiments with the accelerator and to Dr C. Chevalier for her assistance with the annealing in $\mathrm{H}_{2}$ ambient.

\section{References}

1 H. Matsui, A. Ikehata and H. Tabata, Appl. Phys. Lett., 2015, 106, 011905.

2 L. Baldassare, E. Sakat, J. Frogerio, A. Samarelli, K. Gallacher, E. Calandrini, G. Isella, M. Ortolani and P. Biagioni, Nano Lett., 2015, 15, 7225-7231.

3 A. Comin and L. Manna, Chem. Soc. Rev., 2014, 43, 39573975.

4 X. Liu and M. T. Swihart, Chem. Soc. Rev., 2014, 43, 39083920.

5 S. Kalusniak, S. Sadofev and F. Hennerberger, Phys. Rev. Lett., 2014, 112, 137401.

6 Y. Yin and A. P. Alivisatos, Nature, 2005, 437, 664-670.

7 E. Della Gaspera, N. W. Duffy, J. van Embden, L. Waddington, L. Bourgeois, J. J. Jasieniak and A. S. R. Chesman, Chem. Commun., 2015, 51, 12369-12372.
8 P. K. Jain, K. Manthiram, J. Engel, S. L. White, J. A. Faucheaux and A. P. Alivisatos, Angew. Chem., Int. Ed., 2013, 52, 13671-13675.

9 X. Ye, D. Reifsnyder Hickey, J. Fei, B. T. Diroll, T. Paik, J. Chen and C. B. Murray, J. Am. Chem. Soc., 2014, 136, 5106-5115.

10 X. Ye, J. Fei, B. T. Diroll, T. Paik, J. Chen and C. B. Murray, J. Am. Chem. Soc., 2014, 136, 11680-11686.

11 J. M. Luther, P. K. Jain, T. Ewers and A. P. Alivisatos, Nat. Mater., 2011, 10, 361-366.

12 D. M. Smyth, Solid State Ionics, 2000, 129, 5-12.

13 R. W. Johns, H. A. Bechtel, E. L. Runnerstrom, A. Agrawal, S. D. Lounis and D. J. Milliron, Nat. Commun., 2016, 7, 11583.

14 S. D. Lounis, E. L. Runnerstrom, A. Bergerud, D. Nordlund and D. J. Milliron, J. Am. Chem. Soc., 2014, 136, 7110-7116.

15 S. D. Lounis, E. L. Runnerstrom, A. Llordes and D. J. Milliron, J. Phys. Chem. Lett., 2014, 5, 1564-1574.

16 D. Demchenko, B. Earles, H. Liu, V. Avrutin, N. Izyumskaya, Ü. Özgür and H. Morkoç, Phys. Rev. B: Condens. Matter Mater. Phys., 2011, 84, 075201.

17 M. Hamza, J. M. Bluet, K. Masenelli-Varlot, B. Canut, O. Boisron, P. Melinon and B. Masenelli, Nanoscale, 2015, 7, 12030-12037.

18 D. Hapiuk, B. Masenelli, K. Masenelli-Varlot, D. Tainoff, O. Boisron, C. Albin and P. Melinon, J. Phys. Chem. C, 2013, 117, 10220-10227.

19 R. Buonsanti, A. Llordes, A. Aloni, B. A. Helms and D. J. Milliron, Nano Lett., 2011, 11, 4706-4710.

20 R. Mendelsberg, G. Garcia, H. Li, L. Manna and D. J. Milliron, J. Phys. Chem. C, 2012, 116, 12226-12231.

21 V. Juvé, M. Cardinal, A. Lombardi, A. Crut, P. Maioli, J. PérezJuste, L. Liz-Marzán, N. Del Fatti and F. Vallée, Nano Lett., 2013, 13, 2234-2240.

22 H. Y. Liu, V. Avrutin, N. Izyumskaya, M. A. Reshchikov, Ü. Ozgur and H. Morkoç, Phys. Status Solidi RRL, 2010, 4, 70-72.

23 J. T. Thienprasert, S. Rujirawat, W. Klysubun, J. N. Duenow, T. J. Coutts, S. B. Zhang, D. C. Look and S. Limpijumnong, Phys. Rev. Lett., 2013, 110, 055502.

24 D. C. Look, T. C. Droubay and S. A. Chambers, Appl. Phys. Lett., 2012, 101, 102101.

25 A. G. Marrani, F. Capriolo, A. Boccia, R. Zanoni and F. Decker, J. Solid State Electrochem., 2014, 18, 505-513.

26 E. Della Gaspera, D. F. Kennedy, J. van Embden, A. S. R. Chesman, T. R. Gengenbach, K. Weber and J. J. Jasieniak, Adv. Funct. Mater., 2015, 25, 7263-7271.

27 B. L. Greenberg, S. Ganguly, J. T. Held, N. J. Kramer, K. A. Mkhoyan, E. S. Aydil and U. R. Kortshagen, Nano Lett. , 2015, 15, 8162-8169.

28 D. C. Look, K. D. Leedy, D. H. Tomich and B. Bayraktaroglu, Appl. Phys. Lett., 2010, 96, 0621102.

29 J. H. Kim, B. D. Ahn, C. H. Lee, K. A. Jeon, H. S. Kang and S. Y. Lee, J. Appl. Phys., 2006, 100, 113515.

30 B. Ayachi, T. Aviles, J.-P. Vilcot and C. Sion, Appl. Surf. Sci., 2016, 366, 53-58. 
31 A. A. Letailleur, S. Y. Grachev, E. Barthel, F. Sondergard, K. Nomenvo, C. Couteau, S. Mc Murtry and G. Lerondel, J. Lumin., 2011, 131, 2646-2651.

32 W. Tang and D. C. Cameron, Thin Solid Films, 1994, 238, 8387.

33 V. Shelke, B. K. Sonawane, M. P. Bhole and D. S. Patil, J. NonCryst. Solids, 2009, 355, 840-843.
34 V. Musat, B. Teixeira, E. Fortunato and R. C. C. Monteiro, Thin Solid Films, 2006, 502, 219-222.

35 B.-Y. Oh, M.-C. Jeong, D.-S. Kim, W. Lee and J.-M. Myoung, J. Cryst. Growth, 2005, 281, 475-480.

36 E. Della Gaspera, M. Bersani, M. Cittadini, M. Gulglielmi, D. Pagani, R. Noriega, S. Mehra, A. Salleo and A. Martucci, J. Am. Chem. Soc., 2013, 135, 3439-3448. 